\title{
RESTRICTION TO SYMMETRIC SUBGROUPS OF UNITARY REPRESENTATIONS OF RANK ONE SEMISIMPLE LIE GROUPS
}

\author{
B. SPEH AND G. ZHANG
}

\begin{abstract}
We consider the spherical complementary series of rank one Lie groups $H_{n}=\mathrm{SO}_{0}(n, 1 ; \mathbb{F})$ for $\mathbb{F}=\mathbb{R}, \mathbb{C}, \mathbb{H}$. We prove that there exist finitely many discrete components in its restriction under the subgroup $H_{n-1}=\mathrm{SO}_{0}(n-1,1 ; \mathbb{F})$. This is proved by imbedding the complementary series into analytic continuation of holomorphic discrete series of $G_{n}=S U(n, 1), S U(n, 1) \times S U(n, 1)$ and $S U(2 n, 2)$ and by the branching of holomorphic representations under the corresponding subgroup $G_{n-1}$.
\end{abstract}

\section{INTRODUCTION}

This paper is a continuation of [22] by Speh-Venkataramana and [26] by Zhang. Consider the complementary series representations $\left(\pi_{\mu}, H\right)$ of the Lie group $H=S O_{0}(n, 1 ; \mathbb{F})$ for $\mathbb{F}=\mathbb{R}, \mathbb{C}, \mathbb{H}$ and its restriction to the subgroup $H_{1}=S O_{0}(n-1,1 ; \mathbb{F})$. It is proved there that for certain parameters $\mu$ the restricted representation has a direct summand which is isomorphic to a complementary series representation of the smaller group $H_{1}$; similar results are proved also for the exceptional Lie group $H=S O_{0}(2,1 ; \mathbb{O}):=$ $F_{4(-20)}$, and $H_{1}=\operatorname{Spin}(8,1)$.

In [22] the complementary series $\left(\pi_{\mu}, H\right)$ of $H=S O_{0}(n, 1 ; \mathbb{R})$ are realized in its non-compact picture, namely as appropriate spaces of distributions on the space $\mathbb{R}^{n-1}$ and it is proved that, roughly speaking, up to the Fourier transform, the trivial extension of distributions on the subspace $\mathbb{R}^{n-2}$ to distributions $\mathbb{R}^{n-1}$ defines an $H_{1}$-intertwining operator and thus determines for proper values of $\mu$ a discrete summand of $\left(\pi_{\mu}, H\right)$.

The general rank one case is treated in [26] using the compact picture realizing the complementary series as certain Sobolev type spaces on the sphere in $\mathbb{F}^{n}$, the discrete components being obtained by restricting the functions to the sub-sphere in $\mathbb{F}^{n-1}$. This requires some detailed estimates for the branching of representations of the maximal compact subgroup $K \subset H$ on the space of spherical harmonics on $\mathbb{F}^{n}$ under the compact subgroup $L \subset H_{1}$.

In the present paper we determine additional discrete summands of the restriction of $\left(\pi_{\mu}, H\right)$ to $H_{\text {! }}$. These are also isomorphic to complementary series representations of $H_{1}$ and we determine explicitly the parameters of these complementary series representations of $H_{1}$. See theorem 5.1 for a precise statement.

Research by B.Speh partially supported by NSF grant DMS-0901024 and research by G. Zhang partially supported by the Swedish Science Council (VR). 
In the proof we shall use a rather different idea than in the previous papers. We consider $H$ respectively $H_{1}$ as subgroups of a larger groups $G$, respectively $G_{1}$ so that we have the following commutative diagram of subgroup inclusions

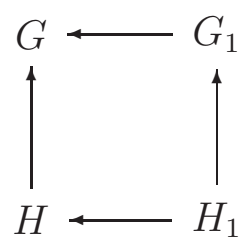

Here we shall consider the Hermitian groups $G=S U(n, 1), S U(n, 1) \times S U(n, 1)$, $S U(2 n, 2)$.

We start with an scalar holomorphic representation $\left(U_{\nu}, G\right)$ of $G$ realized on a Hilbert space of holomorphic functions on the unit ball $G / K$, and consider its branching along the diagram. Denote $\left(U_{\nu}^{b}, G_{1}\right)$ the scalar holomorphic representations of $G_{1}$. The restriction of $\left(U_{\nu}, G\right)$ to $G_{1}$ is a direct sum of unitary representations and the branching rules are obtained by normal holomorphic differentiation $\mathcal{D}^{k}$ of degree $k$ along the submanifold manifold $G_{1} / K_{1}$ in $G / K$. See also [14] for an abstract study. The representations appearing in the restriction will be also holomorphic representations and we are interested in the scalar ones $\left(U_{\nu^{\prime}}, G_{1}\right)$. The branching of $\left(U_{\nu}, G\right)$ under $H$ involves more analytic issues, and has been studied extensively [19, 23, 27, 28, 29]. In particular it is proved that the complementary series $\left(\pi_{\mu}, H\right)$ appears in $\left(U_{\nu}, G\right)$ for certain pairs $(\mu, \nu)$. In the compactpicture of complementary series as distributional spaces on the boundary $S=H / M A N$ of the unit ball $H / L$ there is an intertwining operator

$$
J_{\nu}:\left(\pi_{\mu}, H\right) \rightarrow\left(U_{\nu}, G\right)
$$

obtained by pairing the distributions on $S$ in the complementary series with the reproducing kernel of the holomorphic representation as a function on $S \times G / K$, resulting in a kind of holomorphic extension of complementary series $\left(\pi_{\mu}, H\right)$ in $\left(U_{\nu}, G\right)$. The same is true also for $\left(\pi_{\mu^{\prime}}, H_{1}\right)$ and $\left(U_{\nu^{\prime}}, G_{1}\right)$. The problem reduces thus to proving the operator $\left(J_{\nu^{\prime}}^{b}\right) * \mathcal{D}^{k} J_{\nu}$ in the diagram

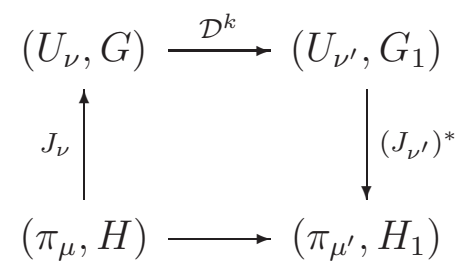

is non-zero for appropriate $\mu$ and $\mu^{\prime}$. This will be done by computing the operator acting on certain vector in $\left(\pi_{\mu}, H\right)$; see Theorem 5.1 below.

It is clear that, compared with the method in [26] where we study boundedness property of the restriction by $K$-type expansion, there are some advantages with the present approach as all the intertwining operators involved have been studied before and there also is flexibility of choosing the degree of differentiation. 
The above procedure of holomorphic extension and differentiation can be applied to general spherical (not necessarily unitary) representations and we can find formally intertwining operators from spherical principal series of $H=S O_{o}(n, 1 ; F)$ to that of $H_{1}=S O_{o}(n-1,1 ; F)$. In the above compact realization of the representations as distribution spaces on the sphere $S$ for $H$ and respectively on the subsphere $S^{b}$ for $H_{1}$, those operators will be polynomials of normal differentiations orthogonal to the totally geodesic submanifold $S^{b} \subset S$ and of horizontal differentiations parallel to $S^{b} \subset S$. The exact formulas can be rather involved. For the real case, $H=S O_{o}(n, 1), H_{1}=S O_{o}(n-1,1)$, those operators have been found by Juhl [12] using computations involving enveloping algebras and Verma modules. See also Kobayashi [15]. In the last section we shall give for $S O_{0}(n, 1)$ a direct proof of our result using these differential intertwining operators in the non-compact realizations.

The results in [22] suggest that similar results should be true for the nonspherical complementary series representations of $S O_{o}(n, 1)$ and more generally for complementary series of all connected semi simple groups of real rank 1.

The branching of complementary series is of interests in automorphic representations [22], [21], [2], [3], [1].

After a preliminary version of this paper was completed we were informed of the preprint [17] where they obtained a complete irreducible decomposition of $\left(\pi_{\mu}, S O(n, 1)\right)$ under the subgroup $S O(n-1,1)$. The case $n=3$ has been obtained earlier in mathematical physics litteratures; see e.g. [18].

Part this work was started during an AIM work "Branching problems in unitary representations", MPIM, Bonn, July 2011. We thank AIM and MPIM for providing a very stimulating environment. We are also grateful to Roger Howe and T. N. Venkataramana for several conversations, and Bent Ørsted for drawing our attention to the work [12].

\section{SPHERICAL REPRESENTATIONS OF CONNECTED SEMI SIMPLE LIE GROUPS OF}

\section{REAL RANK 1}

We recall very briefly some known results and fix some notation. Let $\mathbb{F}=\mathbb{R}, \mathbb{C}, \mathbb{H}$ be the real, complex and quaternionic numbers of real dimension $\iota=1,2,4$. Let $\mathbb{F}^{p, q}:=$ $\mathbb{F}^{p+q}$ be equipped with the quadratic form $\left|x_{1}\right|^{2}+\cdots+\left|x_{p}\right|^{2}-\left|x_{p+1}\right|^{2}-\cdots\left|x_{p+q}\right|^{2}$ for signature $(\iota p, \iota q)$. Denote $S O_{0}(p, q ; \mathbb{F})$ the connected component of group of $\mathbb{F}$-linear transformations on $\mathbb{F}^{p, q}$ preserving the form and having determinant 1 , with $\mathbb{F}$ acting on the right. In the usual notation it is equal to $S O_{0}(p, q), S U(p, q), S p(p, q)$ respectively. Elements in $S O_{0}(p, q ; \mathbb{F})$ and its Lie algebra $\mathfrak{s o}(p, q ; \mathbb{F})$ will be written as $(p+q) \times(p+q)$ block $\mathbb{F}$-matrices

$$
\left[\begin{array}{ll}
a_{1} & a_{2} \\
a_{3} & a_{4}
\end{array}\right]
$$

where $a_{1}, a_{2}, a_{3}, a_{4}$ are of size $p \times p, p \times q, q \times p, q \times q$ respectively. We shall consider subgroups $S O_{0}(r, s ; \mathbb{F})$ in $S O_{0}(p, q ; \mathbb{F}), r \leq p, s \leq q$, and fix the realizations as follows. 
Write $\mathbb{F}^{p, q}=\mathbb{F}^{r} \oplus \mathbb{F}^{p-r} \oplus \mathbb{F}^{p} \oplus \mathbb{F}^{q-s}$. The group $S O_{0}(r, s ; \mathbb{F})$ consists of elements in $S O_{0}(p, q ; \mathbb{F})$ fixing all vectors $\mathbb{F}^{p-r} \oplus \mathbb{F}^{q-s}$ in the above decomposition. The Lie algebra $\mathfrak{s o}(r, s ; \mathbb{F})$ consists of all elements in $\mathfrak{s o}(p, q ; \mathbb{F})$ with the entries $a_{j}$ being diagonal matrix

$$
a_{j}=\left[\begin{array}{cc}
b_{j} & 0 \\
0 & 0
\end{array}\right],
$$

with the obvious sizes of the blocks. The Riemannian symmetric space of $S O_{0}(p, q ; \mathbb{F})$ will be realized as the matrix ball in $M_{p, q}(\mathbb{F})$,

$$
S O_{0}(p, q ; \mathbb{F}) / S(S O(p ; \mathbb{F}) \times S O(q ; \mathbb{F}))=\left\{z \in M_{p, q}(\mathbb{F}) ; z z^{*}<I\right\} .
$$

The above identification defines a totally geodesic submanifold

$$
S O_{0}(r, s ; \mathbb{F}) / S(S O(r ; \mathbb{F}) \times S O(s ; \mathbb{F})) \subset S O_{0}(p, q ; \mathbb{F}) / S(S O(p ; \mathbb{F}) \times S O(q ; \mathbb{F}))
$$

We fix now $H=S O_{0}(n, 1 ; \mathbb{F})$. The maximal compact subgroup $L$ is equal to $S O(n)$, $S(U(n) \times U(1)), S p(n) \times S p(1)$ respectively and and $H / L$ is a Riemannian symmetric space of rank one.

Let $\mathfrak{h}=\mathfrak{l}+\mathfrak{q}$ be the corresponding Cartan decomposition of the Lie algebra $\mathfrak{h}$. Elements in $\mathfrak{q}$ are of the form

$$
\left[\begin{array}{cc}
0 & v \\
v^{*} & 0
\end{array}\right]
$$

with $v \in \mathbb{F}^{n}$. Here $v^{*}=\overline{v^{T}}$ the conjugate transpose where $\bar{a}$ is the conjugation in $\mathbb{F}$. We shall thus identify $\mathfrak{q}$ with $\mathbb{F}^{n}$. We fix

$$
H_{0}=e_{1} \in \mathfrak{q}=\mathbb{F}^{n}, e_{1}^{T}=[1, \cdots, 0]
$$

for $\mathbb{F}=\mathbb{R}$ or $\mathbb{C}$; for $\mathbb{F}=\mathbb{H}$ we let $H_{0}$ be as in [26] so that $\operatorname{Ad}\left(H_{0}\right)$ has eigenvalues $\pm 2, \pm 1,0$. Then $\mathfrak{a}:=\mathbb{R} H_{0}$ is a maximal abelian subspace of $\mathfrak{q}$. The root space decomposition of $\mathfrak{h}$ under $H_{0}$ is

$$
\mathfrak{h}=\mathfrak{n}_{-2}+\mathfrak{n}_{-1}+(\mathfrak{a}+\mathfrak{m})+\mathfrak{n}_{1}+\mathfrak{n}_{2}
$$

with $\pm 2, \pm 1,0$, if $\mathbb{F}=\mathbb{C}, \mathbb{H}$, and with the convention that $\mathfrak{n}_{2}=0$ if $\mathbb{F}=\mathbb{R}$. Here $\mathfrak{m} \subset \mathfrak{l}$ is the zero root space. We denote by $\mathfrak{n}=\mathfrak{n}_{1} \oplus \mathfrak{n}_{2}$ the sum of the positive root spaces. Then $\mathfrak{m}+\mathfrak{a}+\mathfrak{n}$ is a maximal parabolic subalgebra of $\mathfrak{h}$.

Denote $M, A, N$ the corresponding subgroups with Lie algebras $\mathfrak{m}, \mathfrak{a}, \mathfrak{n}$. Then $M=$ $S O(n-1), S U(n-1)$, respectively $S p(n-1) \times S p(1)$ and $M A N$ is a maximal parabolic subgroup of $H$. We normalize the $K$-invariant norm on $\mathfrak{p}$ so that $H_{0}$ is a unit vector. The space $S=K / M$ is then the unit sphere in $\mathfrak{p}$ with $M$ the isotropic subgroup of $H_{0} \in S$.

Let $\rho=\rho_{H}$ be the half sum of positive roots. Then

$$
\rho\left(H_{0}\right)= \begin{cases}\frac{n-1}{2}, & \mathbb{F}=\mathbb{R} \\ n, & \mathbb{F}=\mathbb{C} \\ 2 n+1, & \mathbb{F}=\mathbb{H}\end{cases}
$$


and we shall identify $\rho=\rho\left(H_{0}\right)$. We let $H_{1} \subset H$ be the the subgroup $S O_{0}(n-1,1, \mathbb{F})$ with the above convention of the notation. Let $\mathfrak{h}_{1}=\mathfrak{l}_{1}+\mathfrak{q}_{1}$ be the Cartan decomposition of $\mathfrak{h}_{1}$ obtained under the Cartan involution on $\mathfrak{h}$ restricted to $\mathfrak{h}_{1}$. The subspace $\mathfrak{a}=\mathbb{R} H_{0} \subset \mathfrak{q}$ is also in $\mathfrak{q}_{1}$, and we have the corresponding root space decomposition

$$
\mathfrak{h}_{1}=\mathfrak{n}_{-2,1}+\mathfrak{n}_{-1,1}+\left(\mathfrak{a}+\mathfrak{m} \cap \mathfrak{l}_{1}\right)+\mathfrak{n}_{1,1}+\mathfrak{n}_{2,1},
$$

with

$$
\mathfrak{n}_{ \pm 2,1}=\mathfrak{n}_{ \pm 2}, \quad \mathfrak{n}_{ \pm 1,1}=\mathbb{F}^{n-2} \subset \mathfrak{n}_{ \pm 1}=\mathbb{F}^{n-1}
$$

and $\mathfrak{n}_{1} / \mathfrak{n}_{1,1}=\mathbb{F}$.

The corresponding subgroup $P_{1}=P \cap H_{1}=\left(L_{1} \cap M\right) A N_{1}$ is a maximal parabolic subgroup of $H_{1}$, where $N_{1}$ is the nilpotent subgroup with Lie algebra $\mathfrak{n}_{1}=\mathfrak{n}_{1,1}+\mathfrak{n}_{2,1}$. The homogeneous space $L / K \cap M$ is a subsphere $S_{1}:=S^{\iota(n-1)-1}$ in the sphere $S:=$ $K / M=S^{\iota n-1}$,

$$
S_{1}=L / K \cap M \subset S=K / M
$$

Here $\iota=1,2$ or 4 .

For $\mu \in \mathbb{C}$ let $\pi_{\mu}$ be the spherical principal series representation of $H$ induced from $M A N$ consisting of measurable functions $f$ on $H$ such that

$$
f\left(g m e^{t H_{0}} n\right)=e^{-\mu t} f(g), m e^{t H_{0}} n \in M A N
$$

and $\left.f\right|_{L} \in L^{2}(L)$. In particular elements $\left.f\right|_{L}$ are invariant under the right action of $M$ and thus $\left.f\right|_{L} \in L^{2}(L / M)=L^{2}(S)$. The group action of $\pi_{\mu}(h)$ of $H$ on $L^{2}(S)$ is

$$
\pi_{\mu}(h) f(u)=|c u+d|^{-\mu} f\left((a u+b)(c u+d)^{-1}\right), \quad h^{-1}=\left[\begin{array}{ll}
a & b \\
c & d
\end{array}\right]
$$

for $f \in L^{2}(S)$. This can be derived for example by using that if

$$
h=\left[\begin{array}{ll}
a & b \\
c & d
\end{array}\right] \in H, u=k \cdot H_{0}=k M A N \in S=L / M .
$$

then

$$
e^{-2 \rho A(h k)}=J_{h}(u)=|c u+d|^{-2 \rho},
$$

as proved in [11, 4]. Here $e^{H(h)}$ is the $A$-component in the Iwasawa decomposition $g=$ $k e^{A(h)} n$ of $H=L A N$ of $H, J_{g}(u)$ is the Jacobian of $g: u \rightarrow g u$ on $S$.

The Lie algebra action $\left(\pi_{\mu}, \mathfrak{h}\right)$ on the space $L^{2}(S)_{L}$ of $L$-finite elements in $L^{2}(S)$ defines a Harish-Chandra module which we denote also denote $\pi_{\mu}$.

If $\mu$ is real and $0<\mu<\rho\left(H_{0}\right)$ provided that $\mathbb{F}=\mathbb{R}, \mathbb{C}$ and $2<\mu<\rho\left(H_{0}\right)$ for $\mathbb{F}=$ $\mathbb{H}$ the representations $\left(L^{2}(S)_{L}, \pi_{\mu}, \mathfrak{h}\right)$ are unitarizable and called complementary series. The unitarization of $\left(L^{2}(S)_{L}, \pi_{\mu}, \mathfrak{h}\right)$ can be done through the Knapp-Stein intertwining operator. Its exact formula will not be used in our paper, but we recall it briefly as it motivates our definition of the $J$-operator in Theorem 4.2. 
Let $\langle x, u\rangle=x_{1} \bar{u}_{1}+\cdots+x_{n} \bar{u}_{n}$ be the $\mathbb{F}$-valued inner product on $\mathbb{F}^{n}$. Let

$$
\Lambda_{\mu} f(x)=C_{\mu} \int_{S} \frac{f(u)}{|1-\langle x, u\rangle|^{\mu}} d u .
$$

We note that the integral is absolutely convergent for $\mu<\rho$ for any bounded function $f$ on $S$. As an operator on $L$-finite elements in $L^{2}(S)$ it has meromorphic continuation in $\mu$ to the complex plane. Then

$$
\Lambda_{2 \rho-\mu}:\left(L^{2}(S)_{L}, \pi_{\mu}\right) \rightarrow\left(L^{2}(S)_{L}, \pi_{2 \rho-\mu}\right)
$$

is a $\mathfrak{h}$-intertwining operator. A $\mathfrak{h}$-invariant Hermitian form on $L^{2}(S)_{L}$ is given by

$$
(f, g)_{\mu}=\left(\Lambda_{2 \rho-\mu} f, g\right) .
$$

The decomposition of $L^{2}(S)$ under $L$ is multiplicity free [11], $L^{2}(S)=\sum_{\tau} W^{\tau}$. In particular the operator $\Lambda_{2 \rho-\mu}$ is diagonal under the decomposition,

$$
\Lambda_{2 \rho-\mu} f=\lambda_{\mu}(\tau) f, \quad \text { for } f \in W^{\tau},
$$

with $\lambda_{\mu}(\tau)$ explicitly found in [11].

Theorem 2.1. [16] If

(1) $\mathbb{F}=\mathbb{R}, 0<\mu<n-1=2 \rho_{H}$;

(2) $\mathbb{F}=\mathbb{C}, 0<\mu<2 n=2 \rho_{H}$;

(3) $\mathbb{F}=\mathbb{H}, 2<\mu<4 n=2 \rho_{H}-2$,

there is a positive definite $\mathfrak{h}$-invariant form on $L^{2}(S)_{L}$ defined on the isotopic component $W^{\tau}$ by

$$
\|w\|_{\mu}^{2}=\lambda_{\mu}(\tau)\|w\|^{2} .
$$

Its completion $\left(\mathcal{C}_{\mu}, \pi_{\mu}, H\right)$ defines an unitary irreducible complementary series representations of $H$.

The unitary representations $\pi_{\mu}$ and $\pi_{2 \rho-\mu}$ are unitarily equivalent. To avoid possible confusions we always assume that $\mu<\rho_{H}$, i.e. $\mu$ is in the left half of the above interval for complementary series.

\section{ANALYTIC CONTINUATION OF HOLOMORPHIC DISCRETE SERIES OF UNITARY GROUPS AND THEIR RESTRICTIONS TO A UNITARY SUBGROUP}

We fix pairs $\left(G, G_{1}\right)$ as follows

(1) $\mathbb{F}=\mathbb{R},\left(G, G_{1}\right)=(S U(n, 1), S U(n-1))$;

(2) $\mathbb{F}=\mathbb{C},\left(G, G_{1}\right)=(S U(n, 1) \times S U(n, 1), S U(n-1,1) \times S U(n-1,1))$;

(3) $\mathbb{F}=\mathbb{H},\left(G, G_{1}\right)=(S U(2 n, 2), S U(2(n-1), 2))$.

In this action we discuss the branching of analytic continuation of the holomorphic discrete series of $G$ under under restriction to $G_{1}$. Results for $\mathbb{F}=\mathbb{C}$, i.e., the tensor product case, are trivial generalization of those for $\mathbb{R}$ and will be treated only very briefly. 
The group $G$ is a Hermitian Lie group and its symmetric space $G / K$ will be realized as a bounded symmetric domain $D$.

More precisely we view the complex matrices $z$ in

$$
M_{2 n, 2}(\mathbb{C})=M_{2,2}(\mathbb{C}) \oplus \cdots \oplus M_{2,2}(\mathbb{C})
$$

as column vectors $z=\left[z_{1}, \cdots, z_{n}\right]^{T}$ with each $z_{j}$ being $2 \times 2$-matrices and define

$$
V= \begin{cases}\mathbb{C}^{n} & \text { Case } \mathbb{R} \\ \mathbb{C}^{n} \oplus \overline{\mathbb{C}^{n}} & \text { Case } \mathbb{C} \\ M_{2 n, 2}(\mathbb{C}) & \text { Case } \mathbb{H}\end{cases}
$$

The group $G$ acts on

$$
D=\left\{z \in V ; z z^{*}<I\right\}
$$

by

$$
g z=(a z+b)(c z+d)^{-1}, g=\left[\begin{array}{ll}
a & b \\
c & d
\end{array}\right] \in G,
$$

in the cases of $\mathbb{R}$ and $\mathbb{H}$, it acts on the second component $\left(z_{1}, z_{2}\right) \in D$ as anti-holomorphic mappings in the case $\mathbb{C}$.

The holomorphic discrete series of $G$ are realized as weighted Bergman spaces of holomorphic functions on $D$ and are determined by their reproducing kernels. Let

$$
h(z, w)= \begin{cases}1-\langle z, w\rangle & \text { Case } \mathbb{R} \\ \left(1-\left\langle z_{1}, w_{1}\right\rangle\right)\left(1-\left\langle w_{2}, z_{2}\right\rangle\right) & \text { Case } \mathbb{C} \\ \operatorname{det}\left(1-w^{*} z\right) & \text { Case } \mathbb{H} .\end{cases}
$$

Note that the $h$-function for $\mathbb{F}=\mathbb{R}$ can also be written as $\operatorname{det}\left(1-w^{*} z\right)$.

When $\nu$ is in the set

$$
\begin{cases}(n, \infty) & \text { Case } \mathbb{R}, \mathbb{C} \\ (2 n+1, \infty) & \text { Case } \mathbb{H}\end{cases}
$$

the kernel $h(z, w)^{-\nu}$ is the reproducing kernel of a weighed Bergman space $\mathcal{H}_{\nu}$ with a probability measure on $D$, i.e. the define discrete series representations of the universal covering of $G$.

The group $G$ acts on $\mathcal{H}_{\nu}$ via

$$
U_{\nu}(g) f(z)=\operatorname{det}(c z+d)^{-\nu} f\left(g^{-1} z\right), \quad g^{-1}=\left[\begin{array}{ll}
a & b \\
c & d
\end{array}\right],
$$

defining a (projective) unitary representation $\left(U_{\nu}, G\right)$; in the case of $\mathbb{C}$ it is the tensor product. The set of all $\nu \geq 0$ for which $h(z, w)^{-\nu}$ is positive definite (also called Wallach set) is well-known for general bounded symmetric domains [7, 6, 20, 25]; it consists of an open interval and discrete points and we shall only need open interval. In our parametrization we have 
Lemma 3.1. The functions $h(z, w)^{-\nu}$ is positive definite if $\nu$ is in the set

$$
\begin{cases}(0, \infty) & \text { Case } \mathbb{R}, \mathbb{C}, \\ (1, \infty) & \text { Case } \mathbb{H} .\end{cases}
$$

and it determines a unitary (projective) representation $\left(U_{\nu}, G\right)$ on a Hilbert space of holomorphic functions on $D$ with reproducing kernel $h(z, w)^{-\nu}$. In particular the representation $\left(U_{\nu}, G\right)$ is unitary and not in the discrete series if $\nu$ is in the set

$$
\begin{cases}(0, n) & \text { Case } \mathbb{R}, \mathbb{C}, \\ (1,2 n+1) & \text { Case } \mathbb{H}\end{cases}
$$

We denote the analytic continuation also by $\left(\mathcal{H}_{\nu}, U_{\nu}, G\right)=\left(\mathcal{H}_{\nu}(D), U_{\nu}, G\right)$. In this paper we are concerned with representations $\left(\mathcal{H}_{\nu}, U_{\nu}, G\right)$ whose parameter $\nu$ is in a subinterval of the above interval, i.e.,it is not a discrete holomorphic representations.

For the case $\mathbb{C}$ with $G=S U(n, 1) \times S U(n, 1)$ we let $\mathcal{H}_{\nu}(D \otimes \bar{D})=\mathcal{H}_{\nu}(D) \otimes \overline{\mathcal{H}_{\nu}(D)}$ and $\left(\mathcal{H}_{\nu}(D \otimes \bar{D}), U_{\nu}, G\right)$ be the tensor product of $\left(U_{\nu} \otimes \overline{U_{\nu}}, S U(n, 1) \times S U(n, 1)\right)$, for $\nu>0$.

To find the branching of $\left(\mathcal{H}_{\nu}, U_{\nu}, G\right)$ under $G_{1}$ we realize $D_{1}=G_{1} / K_{1}$ as a submanifold of $D=G / K$ as in $\S 2$. For any polynomial $p$ we denote $\partial_{p}$ the corresponding differential operator. Let

$$
Q= \begin{cases}z_{n}, & \text { Case } \mathbb{R} \\ z_{n} \bar{w}_{n}, & \text { Case } \mathbb{C} \\ \operatorname{det}\left(z_{n}\right), & \text { Case } \mathbb{H} .\end{cases}
$$

The polynomial $Q$ on $V$, as tangent space of $D=G / K$, is invariant under the subgroup $K_{1} \subset K$.

Theorem 3.2. Suppose that $\nu$ satisfies the assumptions of 3.1.

(1) The restriction of of $\left(U_{\nu}, G\right)$ under $G_{1}$ is a discrete sum of holomorphic representations of $G_{1}$.

(2) The holomorphic representation $\left(U_{\nu+j}, G_{1}\right)$ for a nonnegative integer $j$, is a discrete summand of representation $\left(U_{\nu}, G\right)$, restricted to $G_{1}$

(3) A non-zero $G_{1}$-intertwining operator from $\left(U_{\nu}, G\right)$ to $\left(U_{\nu+j}, G_{1}\right)$ is given by

$$
\mathcal{D}^{j}:\left.f \rightarrow \partial_{Q^{j}} f\right|_{D_{1}} .
$$

Proof. Since $\left(U_{\nu}, G\right)$ is a highest weight module the first assertion follows from [14]. Following the ideas in [9] to prove the second and third assertion we realize in the case $\mathbb{R}$ the group $G_{1} \subset G$ acts on $\mathcal{H}_{\nu}$ via $U_{\nu}(g) f\left(z^{\prime}, z_{n}\right)=\left(c z^{\prime}+d\right)^{-\nu} f\left(\left(a z^{\prime}+b\right)\left(c z^{\prime}+d\right)^{-1}, z_{n}\left(c z^{\prime}+d\right)^{-1}\right), \quad g^{-1}=\left[\begin{array}{ll}a & b \\ c & d\end{array}\right] \in G_{1}$. Thus $\left(\partial_{n}^{j}\right)_{z_{n}=0}$ is an intertwining operator $\partial_{n}^{j}\left(U_{\nu}(g) f\right)\left(z^{\prime}, 0\right)=\left(c z^{\prime}+d\right)^{-\nu-j}\left(\partial_{n}^{j} f\right)\left(\left(a z^{\prime}+b\right)\left(c z^{\prime}+d\right)^{-1}, 0\right)=\pi_{\nu+j}(g)\left(\partial_{n}^{j} f(\cdot, 0)\right)$. 
The case $\mathbb{C}$ follows trivially. In the case $\mathbb{H}$ the intertwining property is a simple consequence of

$$
\operatorname{det}\left(\partial_{n}\right)^{j}\left(f\left(A z_{n}\right)\right)=\operatorname{det}(A)^{j}\left(\operatorname{det}\left(\partial_{n}\right)^{j} f\right)\left(A z_{n}\right)
$$

for any function $f$ on $M_{2,2}(\mathbb{C})$.

4. The branching of $\left(U_{\nu}, G\right)$ Under $H$. The APPEARANCE OF The COMPLEMENTARY SERIES $\left(\pi_{\mu}, H\right)$

We consider $H \subset G, H_{1} \subset G_{1}$, written as $\left(H, H_{1}\right) \subset\left(G, G_{1}\right)$, where $\left(H, H_{1}\right)=\left(S O_{0}(n, 1 ; \mathbb{F}), S O_{0}(n-1,1 ; \mathbb{F})\right)$

- If $\mathbb{F}=\mathbb{R}$ then $\left(H, H_{1}\right) \subset\left(G, G_{1}\right)$ is the fix point set of the unitary groups under complex conjugation.

- If $\mathbb{F}=\mathbb{C}$ the subgroup pair $\left(H, H_{1}\right) \subset\left(G, G_{1}\right)$ is the diagonally embedded.

- If $\mathbb{F}=\mathbb{H}$ we first identify the quaternions $\mathbb{H}$ as the real form

$$
\mathbb{H}=\left\{\left[\begin{array}{cc}
a+i b & c+i d \\
-c+i d & a-i b
\end{array}\right] \subset M_{2,2}(\mathbb{C})\right.
$$

in $M_{2,2}(\mathbb{C})$, i.e. $\mathbb{F}=\mathbb{H}$ is the set of elements in $M_{2,2}(\mathbb{C})$ fixed by the real involution $J: z=x+i y \rightarrow x-i y$ in $M_{2,2}(\mathbb{C})$. Observe that under this identification

$$
\operatorname{det} \mathbb{H}=|\mathbb{H}|^{2} \text {. }
$$

The involution defines a corresponding $J$ on $M_{2+2 n, 2+2 n}(\mathbb{C})$. The group $H=$ $S p(n, 1)$ is then the realized as the set of elements in $S U(2 n, 2) \subset M_{2+2 n, 2+2 n}(\mathbb{C})$ fixed by $J$.

In this notation the complex-valued polynomial det $: M_{2,2}(\mathbb{C}) \rightarrow \mathbb{C}$ is the unique $S p(1) \times S p(1)$-invariant holomorphic polynomial of degree 2 . The complex matrices $z$ in $M_{2 n, 2}(\mathbb{C})$ are then complexified quaternions column vectors $z=\left[z_{1}, \cdots, z_{n}\right]^{T}$. Consider the vectors $z=\left[z_{1}, \cdots, z_{n}\right]^{T}$ with quaternionic entries, i.e., in the real space $\mathbb{H}^{n}$. Then

$$
p(z)=\operatorname{det} z_{1}+\cdots+\operatorname{det} z_{n}=\left|z_{1}\right|^{2}+\cdots+\left|z_{n}\right|^{2}=\|z\|^{2},
$$

is invariant under $S p(n) \times S p(1)$ with $S p(n)$ acting on the left and $S p(1)$ on the right. Thus the holomorphic polynomial $p(z)=\operatorname{det} z_{1}+\cdots+\operatorname{det} z_{n}$ is also invariant on the complexification $M_{2,2}(\mathbb{C})$ since $\mathbb{H}^{n}$ is a totally real subspace in $M_{2,2}(\mathbb{C})$.

The main result in the section is

Theorem 4.1. Every complementary series $\left(\pi_{\mu}, H\right), \mu<\rho_{H}$, of $H$ is a direct summand of a holomorphic representation of $G$. 
To obtain the precise formulas for the branching we now recall the branching of $\left(\mathcal{H}_{\nu}, U_{\nu}, G\right)$ under $H$. It has been studied in [23, 27, 29]; see also [19] for some general higher rank cases. Let $X=H / L$ be the symmetric space of $H$ as in $\S 2$. The identification of $H$ with a subgroup in $G$ induces an imbedding of $X$ in $D=G / K$ as a totally real totally geodesic submanifold.

Let $R: \mathcal{H}_{\nu} \rightarrow C^{\infty}(X)$ be the restriction

$$
R F(x)=\left\{\begin{array}{l}
F(x), x \in X \\
0 \text { otherwise }
\end{array}\right.
$$

Define the weighted restriction $R_{\nu}: \mathcal{H}_{\nu} \rightarrow C^{\infty}(X)$ by

$$
R_{\nu} F(x)=(1-\langle x, x\rangle)^{\lambda} R F(x)=(1-\langle x, x\rangle)^{\lambda} F(x), x \in X \subset D,
$$

where

$$
\lambda= \begin{cases}\frac{\nu}{2}, & \mathbb{F}=\mathbb{R} \\ \nu, & \mathbb{F}=\mathbb{C} \\ \nu, & \mathbb{F}=\mathbb{H} .\end{cases}
$$

The functions in $\left(\mathcal{H}_{\nu}, G\right)$ are holomorphic and thus smooth so $R$ and $R_{\nu}$ are well-defined. $R_{\nu}$ is an $H$-intertwining operator

$$
R_{\nu}:\left(\mathcal{H}_{\nu}, G\right) \rightarrow\left(C^{\infty}(X), H\right)
$$

with the regular action of $H$ on $C^{\infty}(X)$.

We denote by $\mathcal{H}_{\nu}(\bar{D})$ the space of holomorphic functions on some neighborhoods of $D$; the group $G$ acts $\mathcal{H}_{\nu}(\bar{D})$ via the action $U_{\nu}$. In particular elements in $\mathcal{H}_{\nu}(\bar{D})$ have smooth restrictions to $S$, where $S$ is the sphere in (2.1), i.e., the boundary of $X$. By abuse of notation we denote this restriction also by $R$. We observe that for

$$
\mu=\left\{\begin{array}{l}
\nu, \text { if } \mathbb{F}=\mathbb{R} \\
2 \nu, \text { if } \mathbb{F}=\mathbb{C}, \mathbb{H},
\end{array}\right.
$$

$R$ is an $H$-intertrwining operator

$$
R:\left.\left(\mathcal{H}_{\nu}(\bar{D}), U_{\nu}, G\right)\right|_{H} \rightarrow\left(C^{\infty}(S), \pi_{\mu}, H\right),
$$

If $\mathbb{F}=\mathbb{R}$ this follows immediately from the definition. For the case $\mathbb{F}=\mathbb{C}$ we have

$$
\begin{aligned}
& R\left(U_{\nu}(h, h) f\right)(s)=\left(U_{\nu}(h, h) f\right)(s) \\
= & (c s+d)^{-\nu} \overline{(c s+d)^{-\nu}} f\left((a s+b)(c s+d)^{-1}, \overline{(a s+b)(c s+d)^{-1}}\right) \\
= & |c s+d|^{-2 \nu} R f\left((a s+b)(c s+d)^{-1}\right),
\end{aligned}
$$

where $h^{-1}$ is as in (2.3). The intertwining relation follows from (2.3). The case of $\mathbb{F}=\mathbb{H}$ is a similar argument using the observation that $\operatorname{det}(q)=|q|^{2}$ for any quaternionic number $q$, with $\operatorname{det}(q)$ being the determinant of the corresponding $2 \times 2$-complex matrix $q$. 
Let $R_{\nu}^{*}$ be the adjoint of $R_{\nu}$ considered as a densely defined operator on $L^{2}(X)=$ $L^{2}(H / L)$ with the $G$-invariant measure $d \iota(x)$. Then $R_{\nu} R_{\nu}^{*}$ is an integral (generally unbounded) operator on $L^{2}(X)$

$$
R_{\nu} R_{\nu}^{*} f(x)=\int_{X} B_{\nu}(x, y) f(y) d \iota(x), \quad B(x, y)=\left(\frac{(1-\langle x, x\rangle)(1-\langle x, x\rangle)}{|(1-\langle x, y\rangle)|^{2}}\right)^{\lambda}
$$

This operator is called Berezin transform [27], and the representations defined by the kernel is called the canonical representation [23]. The spectral decomposition of $R_{\nu} R_{\nu}^{*}$ is given in [23]. As a consequence we get the branching of $\left(U_{\nu}, G\right)$ under $H$. There are finitely many discrete components and we shall need the first one (compared in terms of their eigenvalues of the Casimir operator) of them.

Theorem 4.2. Consider the holomorphic representaton $\left(\mathcal{H}_{\nu}(\bar{D}), U_{\nu}, G\right)$. Let

$$
\mu= \begin{cases}\nu, \nu \in\left(0, \rho_{H}\right)=\left(0, \frac{n-1}{2}\right), & \mathbb{F}=\mathbb{R} \\ 2 \nu, \nu \in\left(0, \frac{\rho_{H}}{2}\right)=\left(0, \frac{n}{2}\right), & \mathbb{F}=\mathbb{C} \\ 2 \nu, \nu \in\left(1, \frac{\rho_{H}}{2}\right)=\left(1, \frac{2 n+1}{2}\right), & \mathbb{F}=\mathbb{H} .\end{cases}
$$

Then the complementary series $\left(\pi_{\mu}, H\right)$ is a discrete summand of the restriction of in $\left(\mathcal{H}_{\nu}(\bar{D}), U_{\nu}, G\right)$ to $H$. Moreover it has multiplicity one in the restriction. In particular the operator

$$
R: \mathcal{H}_{\nu}(\bar{D}) \rightarrow C^{\infty}(S)
$$

extends a $H$-invariant bounded operator from $\mathcal{H}_{\nu}$ onto $\pi_{\mu}$.

Note that the relation between the parameters $\nu$ and $\mu$ is determined by the intertwining property (4.1).

We shall need concrete constructions of the intertwining operators. The following result can be derived from [29, Theorem 5.2], where general higher cases are treated. We present the construction and include a proof of the theorem here in the rank one case.

Theorem 4.3. Let $\nu$ be as in Theorem 4.1. Identifying the dual space of $\pi_{\mu}$ with $\pi_{2 \rho-\mu}$ via the standard $G$-invariant paring in $L^{2}(S)$,

$$
(f, g)=\int_{S} f(x) \overline{g(x)} d x
$$

the dual $J_{\nu}=R^{*}$ of $R: \mathcal{H}_{\nu} \rightarrow \pi_{\mu}$ is given by

$$
J_{\nu}:\left.\left(\pi_{2 \rho-\mu}, H\right) \rightarrow\left(U_{\nu}, G\right)\right|_{H}, \quad J_{\nu} f(z)=\int_{S} \frac{f(s)}{h(z, s)^{\nu}} d s, z \in D^{\prime}
$$

The operator $J_{\nu}$ is defined as an integral operator with kernel $h(z, s)^{\nu}$, the reproducing kernel of $H_{\nu}(D)$. We need its expansion [6] into $K$-types of of $H_{\nu}(D)$; for the cases of $\mathbb{F}=\mathbb{R}, \mathbb{C}$ it is just the binomial expansion. 
Let $\mathcal{P}(V)$ be the space of polynomials on $V$. It has [7, 6] an irreducible decomposition under $K$, known as Hua-Schmid decomposition

$$
\mathcal{P}(V)= \begin{cases}\sum_{m=0}^{\infty} \mathcal{P}_{m}\left(\mathbb{C}^{n}\right) & \text { Case } \mathbb{R} \\ \sum_{m_{1}, m_{2}=0}^{\infty} \mathcal{P}_{m}\left(\mathbb{C}^{n}\right) \otimes \overline{P_{m}\left(\mathbb{C}^{n}\right)} & \text { Case } \mathbb{C} \\ \sum_{\underline{\mathbf{m}}=\left(m_{1}, m_{2}\right), m_{1} \geq m_{2} \geq 0} \mathcal{P}_{\underline{\mathbf{m}}} & \text { Case } \mathbb{H}\end{cases}
$$

where $\mathcal{P}_{m}\left(\mathbb{C}^{n}\right)$ is the space of polynomials of degree $m$, and for the case $\mathbb{H}$ the space $\mathcal{P}_{\underline{\mathbf{m}}}$ is an irreducible representation of $K$ generated by $z_{11}^{m_{1}-m_{2}} \operatorname{det}\left(z_{1}\right)^{m_{2}}$ for $z=\left[z_{1}, \cdots, z_{n}\right]^{T} \in$ $V$ with $z_{1} \in M_{2,2}(\mathbb{C})$ (of highest weight $-m_{1} \gamma_{1}-m_{2} \gamma_{2}$ with $\gamma_{1}, \gamma_{2}$ being the HarishChandra roots).

Denote $(\alpha)_{m}=\prod_{j=0}^{m-1}(\alpha+j)$ and $(\alpha)_{\underline{\mathbf{m}}}=\prod_{j=0}^{m_{1}-1}(\alpha+j)(\alpha-1+j)$ the Pochammer symbol. Let $K_{m}(z, w)$ and $K_{\underline{\mathbf{m}}}(z, w)$ be the reproducing kernel of the irreducible spaces $\mathcal{P}_{m}$ and $\mathcal{P}_{\underline{\mathbf{m}}}$ with the Fock space norm. We have then

$$
K_{m}(z, w)=\frac{1}{m !}\langle z, w\rangle^{m}
$$

in Case $\mathbb{R}$ and

$$
K_{\underline{\mathbf{m}}}(z, w)=\frac{d(\underline{\mathbf{m}})}{(2 n)_{\underline{\mathbf{m}}}} \frac{\operatorname{tr}_{m_{1}-m_{2}}\left(w^{*} z\right)}{m_{1}-m_{2}+1} \operatorname{det}\left(w^{*} z\right)^{m_{2}}
$$

in Case $\mathbb{H}$, the first formula is elementary whereas the second can be obtained from [29. Lemma 3.1]. The Faraut-Koranyi expansion is then the binomial series

$$
h(z, w)^{-\nu}=\sum_{m=0}^{\infty}(\nu)_{m} K_{m}(z, w), \quad K_{m}(z, w)=\frac{1}{m !}\langle z, w\rangle^{m}
$$

for $\mathbb{F}=\mathbb{R}$, and

$$
h(z, w)^{-\nu}=\sum_{\underline{\mathbf{m}} \geq 0}^{\infty}(\nu)_{\underline{\mathbf{m}}} K_{\underline{\mathbf{m}}}(z, w),
$$

if $\mathbb{F}=\mathbb{H}$. Equivalently this can be rephrased as

$$
\|f\|_{\mathcal{F}}^{2}=(\nu)_{\underline{\mathbf{m}}}\|f\|_{\nu}^{2}
$$

The case $\mathbb{F}=\mathbb{C}$ is deduced from the real case.

We can now sketch the proof of the theorem.

Proof. The formula for $J_{\nu}$ is obtained using the reproducing kernel property. Since $\left(\pi_{2 \rho-\mu}, H\right)$ is unitary and irreducible we need only to check that $J_{\nu}$ maps the constant function 1 in $\left(\pi_{2 \rho-\mu}, H\right)$ into a non-zero element in $\left(U_{\nu}, G\right)$, namely we have to prove that

$$
F(z):=J_{\nu} 1=\int_{S} \frac{1}{h(z, s)^{\nu}} d s, z \in D
$$

is in $\left(U_{\nu}, G\right)$. 
$\mathbb{F}=\mathbb{R}$. Using the power series expansion of $h(z, s)^{-\nu}$ we find

$$
F(z)=\sum_{m} \frac{(\nu)_{m}}{m !} \int_{S}\langle z, s\rangle^{m} d s
$$

Clearly the integral $\int_{S}\langle z, s\rangle^{m} d s$ is zero unless $m=2 l$ is even, in which case it is a constant multiple of the polynomial $q(z)^{l}:=\left(z_{1}^{2}+\cdots+z_{n}^{2}\right)^{l}$. The constant can be evaluated at $z=e_{1}$, namely

$$
\begin{aligned}
\int_{S}\langle z, s\rangle^{2 l} d s & =q(z)^{l} \int_{S} s_{1}^{2 l} d s=C_{n} B\left(l+\frac{1}{2}, \frac{n-1}{2}\right) q(z)^{l} \\
& =C_{n} B\left(l+\frac{1}{2}, \frac{n-1}{2}\right) q(z)^{l}=C_{n}^{\prime} \frac{\Gamma\left(l+\frac{1}{2}\right)}{\Gamma\left(l+\frac{n}{2}\right)} q(z)^{l}
\end{aligned}
$$

where $C_{n}$ and $C_{n}^{\prime}$ are constants depending only on $n$. Thus

$$
F(z)=C_{n}^{\prime} \sum_{l} \frac{(\nu)_{2 l}}{(2 l) !} \frac{\Gamma\left(l+\frac{1}{2}\right)}{\Gamma\left(l+\frac{n}{2}\right)} q(z)^{l}
$$

and the membership of $F$ in $\left(U_{\nu}, G\right)$ is determined by the convergence of square norm

$$
\|F\|_{\nu}^{2}=C_{n}^{\prime 2} \sum_{l}\left(\frac{(\nu)_{2 l}}{(2 l) !} \frac{\Gamma\left(l+\frac{1}{2}\right)}{\Gamma\left(l+\frac{n}{2}\right)}\right)^{2}\left\|q^{l}\right\|_{\nu}^{2}=C_{n}^{\prime 2} \sum_{l}\left(\frac{(\nu)_{2 l}}{(2 l) !} \frac{\Gamma\left(l+\frac{1}{2}\right)}{\Gamma\left(l+\frac{n}{2}\right)}\right)^{2} \frac{1}{(\nu)_{2 l}}\left\|q^{l}\right\|_{\mathcal{F}}^{2}
$$

The square norm $\left\|q^{l}\right\|_{\mathcal{F}}^{2}$ is

$$
\left\|q^{l}\right\|_{\mathcal{F}}^{2}=2^{2 l} l !\left(\frac{n}{2}\right)_{l}=\frac{\Gamma\left(\frac{1}{2}\right)(2 l) !}{\Gamma\left(\frac{1}{2}+l\right)}\left(\frac{n}{2}\right)_{l}
$$

Simplifying we find

$$
\|F\|_{\nu}^{2}=C_{n}^{\prime \prime} \sum_{l}\left(\frac{(\nu)_{2 l}}{(2 l) !}\right)\left(\frac{\Gamma\left(l+\frac{1}{2}\right)}{\Gamma\left(l+\frac{n}{2}\right)}\right) \sim \sum_{l \geq 1} \frac{1}{l^{-\nu+\frac{n}{2}+\frac{1}{2}}}
$$

which is convergent if and only $\nu<\frac{n-1}{2}=\rho$.

$\mathbb{F}=\mathbb{C}$. This case is studied in [5] where a construction for all discrete components in the branching of $\left.\left(U_{\mu}, G\right)\right|_{H}$ is obtained. The function $F$ above is

$$
F(z, w)=\sum_{m=0}^{\infty} \frac{(\nu)_{m}(\nu)_{m}}{(n)_{m}} \frac{1}{m !}\langle z, w\rangle^{m}, \quad(z, w) \in G / K=B \times B
$$

and

$$
\|F\|_{\nu}^{2}=\sum_{m=0}^{\infty}\left(\frac{(\nu)_{m}(\nu)_{m}}{(n)_{m}}\right)^{2} \frac{\operatorname{dim}\left(\mathcal{P}_{m}\right)}{(\nu)_{m}^{2}}
$$

where $\operatorname{dim}\left(\mathcal{P}_{m}\right) \sim m^{n-1}$ is the dimension of the polynomial space $P_{m}\left(\mathbb{C}^{n}\right)$. Here we have used (4.3) applied to the tensor product. Each term is

$$
\frac{(\nu)_{m}^{2}}{(n)_{m}^{2}} \operatorname{dim}\left(\mathcal{P}_{m}\right) \sim \frac{1}{m^{2 n-2 \nu}} m^{n-1}=\frac{1}{m^{n+1-2 \nu}}
$$

and the series is convergent if an only if $2 \nu<n=\rho_{\mathfrak{s u}(n, 1)}$. 
$\mathbb{F}=\mathbb{H}$. Similarly $F(z)=\sum_{\underline{\mathbf{m}}}(\nu)_{\underline{\mathbf{m}}} \int_{S} K_{\underline{\mathbf{m}}}(z, s) d s$, and the polynomial $\int_{S} K_{\underline{\mathbf{m}}}(z, s) d s$ is an $L$-invariant polynomial in $\overline{\mathcal{P}}_{\underline{\mathbf{m}}}$. It is proved in [29, Lemma 3.3] that this space is zero unless $\underline{\mathbf{m}}=(m, m)$, in which case it is one-dimensional, namely given by the polynomial $q(z)^{m}=\left(\operatorname{det} z_{1}+\cdots+\operatorname{det} z_{n}\right)^{m}$.

$$
\int_{S} K_{\underline{\mathbf{m}}}(z, s) d s=C_{\underline{\mathbf{m}}} q(z)^{m}
$$

We evaluate it at $z=\left[I_{2}, \cdots, 0\right]^{T}$. The left hand side is

$$
\begin{aligned}
\int_{S} \operatorname{det}\left(s_{1}\right)^{m} d s=\int_{S}\left|s_{1}\right|^{2 m} d s & =C \int_{\mathbb{H}}|y|^{2 m}\left(1-|y|^{2}\right)^{2 n-3} d y \\
& =C^{\prime} \int_{0}^{1} r^{2 m}\left(1-r^{2}\right)^{2 n-3} r^{3} d r d y \\
& =C^{\prime \prime} \frac{(m+1) !}{(2 n-1)_{m}}
\end{aligned}
$$

with a normalization constant $C$ depending only only on $n$. The square norm of $F$ in $\left(U_{\mu}, G\right)$ is

$$
\|F\|_{\nu}^{2}=C \sum_{\underline{\mathbf{m}}=(m, m)}(\nu)_{\underline{\mathbf{m}}}^{2}\left(\frac{(m+1) !}{(2 n-1)_{m}}\right)^{2}\left\|q^{m}\right\|_{\mathcal{F}}^{2}
$$

The Fock space norm $\left\|q^{m}\right\|_{\mathcal{F}}^{2}$ of $p^{m}$ can be computed by elementary differentiation. We have the following (Caley-Capelli type) identity

$$
q(\partial) q^{m}=m(m-1+2 n) q^{m-1} .
$$

from which it follows

$$
\left\|q^{m}\right\|_{\mathcal{F}}^{2}=m !(2 n)_{m}
$$

The square norm of

$$
\|F\|_{\nu}^{2} \sim \sum_{m=1}^{\infty} \frac{1}{m^{2 n+2 n-1-(2 n+2 \nu-1)}}
$$

which is convergent precisely when $2 \nu<2 n+1=\rho_{\mathfrak{s p}(n, 1)}$.

\section{DiscRETE SUMMANDS IN THE RESTRICTION OF $\left(\pi_{\nu}, H\right)$ TO THE SUBGROUP $H_{1}$}

We prove now our main theorem of this paper.

\section{Theorem 5.1.}

A.) Assume that $\mathbb{F}=\mathbb{R}$ and $0<\mu<\rho_{H}$. If $\mu+2 j<\rho_{H_{1}} j \geq 0$, then $\left(\pi_{\mu+2 j}, H_{1}\right)$ is $a$ subrepresentation of the restriction of $\left(\pi_{\mu}, H\right)$ to $H_{1}$.

B.) Assume that $\mathbb{F}=\mathbb{C}$ and $0<\mu<\rho_{H}$. If $\mu+2 j<\rho_{H_{1}} j \geq 0$, then $\left(\pi_{\mu+2 j}, H_{1}\right)$ is a subrepresentation of the restriction of $\left(\pi_{\mu}, H\right)$ to $H_{1}$.

C.) Assume that $\mathbb{F}=\mathbb{H}$ and $2<\mu<\rho_{H}$. If $\mu+2 j<\rho_{H_{1}} j \geq 0$, then $\left(\pi_{\mu+2 j}, H_{1}\right)$ is a subrepresentation of the restriction of $\left(\pi_{\mu}, H\right)$ to $H_{1}$. 
Actually we will prove in the next section that for $\mathbb{F}=\mathbb{R}, 0<\mu<\rho_{H}, \mu+j<\rho_{H_{1}}$ $j \geq 0$, then $\left(\pi_{\mu+j}, H_{1}\right)$ is a subrepresentation of the restriction of $\left(\pi_{\mu}, H\right)$ to $H_{1}$. Our proof below uses explicit differentiations of $\partial_{z_{n}^{j^{\prime}}}$ on $\left(z_{1}^{2}+\cdots+z_{n-1}^{2}+z_{n}^{2}\right)^{m}$ evaluated at $z_{n}=0$ which gives trivial result if $j^{\prime}$ is odd. Presumably the same proof will also work for odd $j^{\prime}$ by replacing the function 1 below by $x_{1}$.

Proof. Recall that $\mu$ is defined as a function of $\nu$ by

$$
\mu= \begin{cases}\nu, \nu \in\left(0, \rho_{H}\right)=\left(0, \frac{n-1}{2}\right), & \mathbb{F}=\mathbb{R} \\ 2 \nu, \nu \in\left(0, \frac{\rho_{H}}{2}\right)=\left(0, \frac{n}{2}\right), & \mathbb{F}=\mathbb{C} \\ 2 \nu, \nu \in\left(1, \frac{\rho_{H}}{2}\right)=\left(2, \frac{2 n+1}{2}\right), & \mathbb{F}=\mathbb{H} .\end{cases}
$$

We consider the operator, recalling $\mathcal{D}^{j}$ in Theorem 3.2,

$$
T_{j}=\left(J_{\nu+j^{\prime}}\right)^{*} \mathcal{D}^{j^{\prime}} J_{\nu}=R \mathcal{D}^{j^{\prime}} J_{\nu}
$$

where $j^{\prime}=2 j$ for $\mathbb{F}=\mathbb{R}$ and $j^{\prime}=j$ for $\mathbb{F}=\mathbb{C}, \mathbb{H}$. (We choose $j^{\prime}=2 j$ for $\mathbb{F}=\mathbb{R}$ to ensure that $\left(T_{j} 1,1\right)$ below is non-zero.) Note that it should be clear from the formula the spaces on which the $J$-operators are defined. The operator $T_{j}$ is bounded and $H_{1}$ intertwining

$$
T_{j}=:\left(\mathcal{C}_{2 \rho_{H}-\nu}, \pi_{2 \rho_{H}-\nu}, H\right) \stackrel{J_{\nu}}{\rightarrow}\left(\mathcal{H}_{\nu}, U_{\nu}, G\right) \stackrel{\mathcal{D}^{j^{\prime}}}{\rightarrow}\left(\mathcal{H}_{\nu+j^{\prime}}, U_{\nu+j^{\prime}}, G_{1}\right) \stackrel{R}{\rightarrow}\left(\mathcal{C}_{\mu\left(j^{\prime}\right)}, \pi_{\mu\left(j^{\prime}\right)}, H_{1}\right)
$$

by Theorems 3.2 and 4.2, where $\mu(j)$ is obtained from $\nu+j^{\prime}$ via the above correspondence $\mu \leftrightarrow \nu$. Composed with the equivalence $\Lambda_{2 \rho_{H}-\nu}$ of $\left(\mathcal{C}_{\nu}, \pi_{\nu}, H\right)$ and $\left(\mathcal{C}_{2 \rho_{H}-\nu}, \pi_{2 \rho_{H}-\nu}, H\right)$ we get a bounded $H_{1}$-intertwining operator

$$
T_{j} \Lambda_{\nu}:\left(\mathcal{C}_{\nu}, \pi_{\nu}, H\right) \rightarrow\left(\mathcal{C}_{\mu(j)}, \pi_{\mu(j)}, H_{1}\right)
$$

which preserves the Hilbert space norms on $H$ and $H_{1}$. Thus we need only to check that $T_{j} 1 \neq 0$. We compute the pairing $\left(T_{j} 1,1\right)$ in Theorem 4.2 .

$$
\left(T_{j} 1,1\right)=\left(\mathcal{D}^{j^{\prime}} J_{\nu} 1, J_{2 \rho_{H_{1}}-\mu(j)} 1\right)_{\mathcal{H}_{\nu+j^{\prime}}}
$$

The functions $J_{\nu} 1$ and $J_{2 \rho_{H_{1}}-\mu(j)} 1$ are found in (4.4) in the proof of Theorem 4.2 (for the bigger group pair $(G, H)$ ). We shall find $\mathcal{D}^{k} J_{\nu} 1$ following the computations there.

$\mathbb{F}=\mathbb{R}$. In this case $\mathcal{D}^{2 j} J_{\nu} 1$ is, apart from a positive normalization constant,

$$
\sum_{l \geq j} \frac{(\nu)_{2 l}}{(2 l) !} \frac{\Gamma\left(l+\frac{1}{2}\right)}{\Gamma\left(l+\frac{n}{2}\right)}(2 j) !\left(\begin{array}{c}
l \\
2 j
\end{array}\right) q\left(z^{\prime}, 0\right)^{l-j}
$$

for

$$
\left(\partial_{n}^{j} q^{l}\right)\left(z^{\prime}, 0\right)=(2 j) !\left(\begin{array}{c}
l \\
2 j
\end{array}\right) q\left(z^{\prime}, 0\right)^{l-j}
$$

whereas $J_{2 \rho_{H_{1}}-\mu(j)} 1$ is

$$
F\left(z^{\prime}\right)=C \sum_{k} \frac{\left(2 \rho_{H_{1}}-\mu(j)\right)_{2 k}}{(2 k) !} \frac{\Gamma\left(k+\frac{1}{2}\right)}{\Gamma\left(k+\frac{n}{2}\right)} q\left(z^{\prime}\right)^{k} .
$$


The inner product $\left(\mathcal{D}^{2 j} J_{\nu} 1, J_{\mu} 1\right)_{\mathcal{H}_{2 \rho_{H_{1}}-\mu(j)}}$, is then, up to a positive constant,

$$
\sum_{l-j=k=0}^{\infty} \frac{(\nu)_{2 l}}{(2 l) !} \frac{\Gamma\left(l+\frac{1}{2}\right)}{\Gamma\left(l+\frac{n}{2}\right)}(2 j) !\left(\begin{array}{c}
l \\
2 j
\end{array}\right) \frac{\left(2 \rho_{H_{1}}-\mu(j)\right)_{2 k}}{(2 k) !} \frac{\Gamma\left(k+\frac{1}{2}\right)}{\Gamma\left(k+\frac{n}{2}\right)}\left\langle q\left(z^{\prime}\right)^{k}, q\left(z^{\prime}\right)^{k}\right\rangle_{\mathcal{H}_{\mu(j)}},
$$

which is a (convergent) sum of positive terms and thus is non-zero.

$\mathbb{F}=\mathbb{C}, \mathbb{H}$. The computations are similar. We need only to note that for the $\mathbb{H}$-case,

$$
\partial_{Q}^{j} q^{m}\left(z^{\prime}, 0\right)=\left(\begin{array}{c}
m \\
j
\end{array}\right)(j+1) ! j ! q\left(z^{\prime}, 0\right)^{m-j}
$$

with the coefficients being also positive.

We note that our approach above can also be used to treat the complementary series for $H=F_{4(-20)}$ [10] under the symmetric subgroup $H_{1}=\operatorname{Spin}(8,1)$ by considering the Hermitian Lie groups $G \supset G_{1}$ with $G=E_{6(-25)}, G_{1}=\operatorname{Spin}(8,2)$. However no more extra discrete component will be discovered except the one obtained using the restriction method in [26], namely the zero degree of differentiation in the above method. This is due to the rather short interval of complementary series of $H=F_{4(-20)}$. More precisely the eligible range of the parameter $\nu$ of holomorphic representations $\left(U_{\nu}, G\right)$ for which discrete components under $H$ do appear is the interval $\left(3, \frac{11}{2}\right)$. Here the lower bound 3 corresponds to the first discrete Wallach reducible point for $G$, and the upper bound corresponds to $\frac{\rho_{H}}{2}$ which is the critical point where the spectral symbol of the Berezin transform $R R^{*}$ in (4.2) has a pole [26, (6.1)]. The same range of $\nu$ for $\left(U_{\nu}, G_{1}\right)$ for the pair $\left(G_{1}, H_{1}\right)=(\operatorname{Spin}(8,2), \operatorname{Spin}(8,1))$ is $\left(3, \frac{7}{2}\right)$. (The $\rho_{H}$ and $\rho_{H_{1}}$ are 11 and 7 with the same normalization of split Cartan in $\mathfrak{h}_{1} \subset \mathfrak{h}$, the half values $\frac{11}{2}$ and $\frac{7}{2}$ in the intervals are due to the double rank of $G$ and $G_{1}$ of that of $H$ and $H_{1}$.) The symmetric domain $D_{1}=G_{1} / K_{1}$ is in $V_{1}=\mathbb{O}_{\mathbb{C}}$ as a subdomain of $D=G / K$ in $\mathbb{O}_{\mathbb{C}}^{2}=V_{1} \oplus V_{2}$. A normal differentiation by any $K_{1}$ invariant polynomial on $V_{2}$ on the subdomain $D_{1}=G_{1} / K_{1}$ will result in intertwining operator from $\left(U_{\nu}, G\right)$ into $\left(U_{\nu^{\prime}}, G_{1}\right)$ with $\nu^{\prime} \geq \nu+1$. But for $\nu>3$ we have $\nu^{\prime} \geq 4>\frac{7}{2}$ which is outside the eligible range $\left(3, \frac{7}{2}\right)$. Presumably this is the only possible discrete complement.

\section{EXPLICIT REALIZATION OF DISCRETE COMPONENTS VIA INTERTWINING DIFFERENTIAL OPERATORS}

For the real case a direct proof of the appearance of the discrete components in the branching can be obtained using the explicit formula for intertwining differential operators [12] for spherical representations of $H$ and $H_{1}$. The operators have an easier expression in the non-compact realization. In this realization our proof is a simple application of the Cauchy-Schwarz inequality, and it also yields an explicit construction of the discrete components.

The complementary series $\left(\mathcal{C}_{\mu}, \pi_{\mu}, H\right), H=S O_{0}(n, 1)$, can be realized as distribution space on $\mathfrak{n}=\mathbb{R}^{n-1}$; see e.g. [22]. Initially it acts on a subspace $C_{\mu}^{\infty}\left(\mathbb{R}^{n-1}\right)$ of $C^{\infty}\left(\mathbb{R}^{n-1}\right)$ 
obtained from the restriction to $\mathbb{R}^{n-1}=N^{-}$of smooth functions on $G$; alternatively it can be obtained by a a Cayley transfrom from $S$ to $\mathbb{R}^{n-1}$ and the $H$-action on the space $C^{\infty}(S)$. The action of $P^{-}=M A N^{-}$on the space $C_{\mu}^{\infty}\left(\mathbb{R}^{n-1}\right)$ is

$$
\pi_{\mu}(m) f(x)=f\left(m^{-1} x\right), \pi_{\mu}(a) f(x)=a^{-\mu} f\left(a^{-1} x\right), \pi_{\mu}(y) f(x)=f(x-y),
$$

for

$$
m \in S O_{0}(n-1), a \in \mathbb{R}^{+}=A, y \in \mathbb{R}^{n-1}=N^{-} .
$$

The Weyl group element $w$ acts as an inversion

$$
\pi_{\mu}(w) f(x)=f\left(-\frac{2 x}{|x|^{2}}\right)\left(\frac{2}{|x|^{2}}\right)^{\mu}
$$

The Knapp-Stein intertwining operator is

$$
f \rightarrow \int_{\mathbb{R}^{n-1}} f(y)|x-y|^{-2(n-1-\mu)} d y
$$

as a meromorphic continuation of integral operators. Let $\Delta_{n-1}=\partial_{1}^{2}+\cdots+\partial_{n-1}^{2}$ be the Laplace operator on $\mathbb{R}^{n-1}$ and $\mathcal{F}$ the Fourier transform. Then $\left(\mathcal{C}_{\mu}, \pi_{\mu}, H\right)$ is the completion of $C_{0}^{\infty}\left(\mathbb{R}^{n-1}\right)$ with the norm

$$
\|f\|_{\mu}^{2}=\left\|\Delta^{\frac{n-1-2 \mu}{2}} f\right\|_{L^{2}}^{2}=\int_{\mathbb{R}^{n-1}}|\mathcal{F} f(\xi)|^{2}|\xi|^{n-1-2 \mu} d \xi
$$

for $0<\mu<\frac{n-1}{2}=\rho$. See e.g. [24, Theorem 2.1], our parameter $\mu$ being their $n-1-\lambda$.

The explicit form of $\pi_{\mu}$ is well-known and will not be used here. Correspondingly the representation $\left(\mathcal{C}_{\mu}, \pi_{\mu}, H_{1}\right)$ is realized on $\mathbb{R}^{n-2}$, considered as the subspace $\{(x, 0) ; x \in$ $\left.\mathbb{R}^{n-2}\right\}$ of $\mathbb{R}^{n-1}$.

We recall the result of Juhl [12, Ch. V] on intertwining differential operators, with our parametrization of the spherical representations.

Theorem 6.1. Let $n>2$ and $\mu \in \mathbb{C}$. There exists an non-zero intertwining $H_{1}$ operator

$$
\mathcal{D}_{\mu, j}:\left(C_{\mu}^{\infty}\left(\mathbb{R}^{n-1}\right), \pi_{\mu}, H\right) \rightarrow\left(C_{\mu+j}^{\infty}\left(\mathbb{R}^{n-2}\right), \pi_{\mu+j}, H_{1}\right)
$$

of the form

$$
\mathcal{D}_{\mu, j} f(y, 0)=\left(\sum_{2 k \leq j} C_{\mu, k} \partial_{n-1}^{j-2 k} \Delta_{n-2}^{k} f\right)(y, 0)
$$

where $C_{\mu, k}$ are constants depending on $\mu, m$ normalized by $C_{\mu, 0}=1$.

We give now an independent proof of Theorem 5.1 for the real case.

Theorem 6.2. Let $n>2,0<\mu<\rho_{H}=\frac{n-1}{2}$ and $\mu+j<\rho_{H_{1}}=\frac{n-2}{2}$. Then the operator $\mathcal{D}_{\mu, j}$ is a non-zero $H_{1}$-invariant bounded operator from $\left(\mathcal{C}_{\mu}, H\right)$ onto $\left(\mathcal{C}_{\mu+j}, H_{1}\right)$. Thus the complemetnary series $\left(\mathcal{C}_{\mu+j}, H_{1}\right)$ appears as a discrete component in the decompostion of $\left(\mathcal{C}_{\mu}, H\right)$ under $H_{1}$. 
Proof. Note that the second claim follows from the first one by a standard argument. Indeed the adjoint $\mathcal{D}_{\mu, j}^{*}$ of $\mathcal{D}_{\mu, j}$ is then a non-zero bounded $H_{1}$-invariant operator from $\left(\mathcal{C}_{\mu+j}, H_{1}\right)$ to $\left(\mathcal{C}_{\mu}, H\right)$. Its polar decomposition defines an $H_{1}$-invariant partial isometry. But since $\left(\mathcal{C}_{\mu+j}, H_{1}\right)$ is irreducible it is an isometry.

We denote $\mathcal{F}^{b}$ the Fourier transform on $\mathbb{R}^{n-2}$. Let $f \in C_{0}^{\infty}\left(\mathbb{R}^{n-1}\right)$ and $g:=D_{\mu, j} f$. We shall prove that

$$
\|g\|_{\mu+j}^{2}:=\int_{\mathbb{R}^{n-2}}|\eta|^{n-2-2(\mu+j)}\left|\mathcal{F}^{b} g(\eta)\right|^{2} d \eta \leq C\|f\|_{\mu}^{2} .
$$

By the Fourier inversion formula we have, writing the dual variable $\xi \in \mathbb{R}^{n-1}$ as $\xi=$ $\left(\eta, \xi_{n-1}\right) \in \mathbb{R}^{n-2} \times \mathbb{R}$

$$
f\left(y, x_{n-1}\right)=C_{n} \int_{\mathbb{R}^{n-1}} \mathcal{F} f\left(\eta, \xi_{n-1}\right) e^{i(y, \eta)+i x_{n-1} \xi_{n-1}} d \eta d \xi_{n-1},
$$

where $C_{n}$ is the normalization constant for the Fourier inversion. Performing the differentiation $\mathcal{D}_{\mu, j}$ evaluated at $(y, 0)$ we get

$$
\begin{aligned}
g(y) & =\mathcal{D}_{\mu, j} f(y, 0)=C_{n} \int_{\mathbb{R}^{n-1}} \mathcal{F} f\left(\eta, \xi_{n-1}\right) e^{i(y, \eta)} E\left(\eta, \xi_{n-1}\right) d \eta d \xi_{n-1} \\
& =C_{n} \int_{\mathbb{R}^{n-2}} e^{i(y, \eta)}\left(\int_{\mathbb{R}} \mathcal{F} f\left(\eta, \xi_{n-1}\right) E\left(\eta, \xi_{n-1}\right) d \xi_{n-1}\right) d \eta
\end{aligned}
$$

where $E(\xi)=E\left(\eta, \xi_{n-1}\right)$ is the Fourier multiplier corresponding to (6.2),

$$
E(\xi)=E\left(\eta, \xi_{n-1}\right)=\sum_{2 k \leq j} C_{\mu, k}\left(-|\eta|^{2}\right)^{k}\left(-\xi_{n-1}^{2}\right)^{j-2 k} .
$$

The Fourier transform $\mathcal{F}^{b} g$ of $g$ is

$$
\mathcal{F}^{b} g(\eta)=C \int_{\mathbb{R}} \mathcal{F} f\left(\eta, \xi_{n-1}\right) E\left(\eta, \xi_{n-1}\right) d \xi_{n-1}=C \int_{\mathbb{R}} \mathcal{F} f(\xi) E(\xi) d \xi_{n-1}
$$

where we have written $\xi=\left(\eta, \xi_{n-1}\right)$. We apply the Cauchy-Schwarz inequality, obtaining

$$
\left|\mathcal{F}^{b} g(\eta)\right|^{2} \leq \int_{\mathbb{R}}|\mathcal{F} f(\xi)|^{2}|\xi|^{n-1-2 \mu} d \xi_{n-1} \int_{\mathbb{R}}|E(\xi)|^{2} \frac{1}{|\xi|^{n-1-2 \mu}} d \xi_{n-1} .
$$

Now the polynomial $E(\xi)$ is a sum of terms of the form $\xi_{n-1}^{j-2 k}|\eta|^{2 k}, 0 \leq 2 k \leq j$, and we apply the triangle inequality to the second integration,

$$
\int_{\mathbb{R}}|E(\xi)|^{2} \frac{1}{|\xi|^{n-1-2 \mu}} d \xi_{n-1} \leq C \sum_{k=0} \int_{\mathbb{R}}\left(|\eta|^{2 k} \xi_{n-1}^{j-2 k}\right)^{2} \frac{1}{|\xi|^{n-1-2 \mu}} d \xi_{n-1} .
$$

The each term can be evaluated by a change of variables, $\xi_{n-1}=|\eta| u$,

$$
\int_{\mathbb{R}}\left(|\eta|^{2 k} \xi_{n-1}^{j-2 k}\right)^{2} \frac{1}{|\xi|^{n-1-2 \mu}} d \xi_{n-1}=\frac{1}{|\eta|^{n-2-2 \mu-2 j}} \int_{\mathbb{R}} \frac{u^{4 k}}{\left(1+u^{2}\right)^{n-1-2 \mu}} d u=C \frac{1}{|\eta|^{n-2-2 \mu-2 j}} .
$$

Here we have used the assumption that $j \leq j_{0}$ so that $n-1-2 \mu-4 k \geq n-1-2 \mu-2 j>1$ and the integration

$$
C=\int_{\mathbb{R}} \frac{u^{4 k}}{\left(1+u^{2}\right)^{n-1-2 \mu}} d u<\infty
$$


is convergent. That is

$$
\int_{\mathbb{R}}|E(\xi)|^{2} \frac{1}{|\xi|^{n-1-2 \mu}} d \xi_{n-1} \leq C \frac{1}{|\eta|^{n-2-2 \mu-2 j}}
$$

and

$$
\left|\mathcal{F}^{b} g(\eta)\right|^{2} \leq C \frac{1}{|\eta|^{n-2-2 \mu-2 j}} \int_{\mathbb{R}}|\mathcal{F} f(\xi)|^{2}|\xi|^{n-1-2 \mu} d \xi_{n-1} .
$$

Integrating over $\eta \in \mathbb{R}^{n-2}$ we find

$$
\|g\|_{\mu+j}^{2}=\int_{\mathbb{R}^{n-2}}\left|\mathcal{F}^{b} g(\eta)\right|^{2}|\eta|^{n-2-2 \mu-2 j} \leq C \int_{\mathbb{R}^{n-2}} \int_{\mathbb{R}}|\mathcal{F} f(\xi)|^{2}|\xi|^{n-1-2 \mu} d \xi=C\|f\|_{\mu}^{2}
$$

completing the proof.

For $j=0$, i.e. $\mathcal{D}_{\mu, 0}$ being the restriction, the above result is proved in [22] by considering the dual map of the restriction. Note the above proof is somewhat simpler.

Note the standard $L^{2}\left(\mathbb{R}^{n-1}\right)$-pairing $f \otimes g \rightarrow(f, g)$ is invariant under $\pi_{\mu} \otimes \pi_{2 \rho-\mu}$ and we may rephrase the above theorem as a realization of the discrete components.

Corollary 6.3. Let $\mu$ and $j$ be as above. The representation $\left(\pi_{n-2-(\mu+j)}, H_{1}\right)$ appears in the branching of $\left(\pi_{n-1-\mu}, H\right)$ under $H_{1}$ and an explicit realization of the subrepresentation is the distribution space

$$
\left\{F ; F\left(y, x_{n-1}\right)=\sum_{2 k \leq j} C_{\mu, k} \partial_{n-1}^{j-2 k} \Delta_{n-2}^{k}\left(f(y) \delta\left(x_{n-1}\right)\right), \quad f \in\left(\pi_{n-2-(\mu+j)}, H_{1}\right)\right\},
$$

where $\delta$ is the Dirac's delta function.

We may also use the version of $\mathcal{D}_{\mu, j}$ in the compact picture and to prove Theorem 6.2 directly using the method in [26]. This involves rather detailed computations of normal differentiation of spherical harmonics. The above proof using Fourier transform is apparently easier.

Finally we study the limit case $\mu=0$ using the operator $\mathcal{D}_{\mu, j}$. Assume $n>2$ is an odd integer. The Harish-Chandra module of $K$-finite elements in $C^{\infty}(S)$ has the constant functions $\mathbb{C}$ as its submodule and the quotient space is unitarizable, with the unitary norm obtained from that of $\mathcal{C}_{\mu}$ by continuation (or residue depending on the normalization); see [11]. We denote the quotient space by $\mathcal{W}_{0}$. Now the norm

$$
\|f\|_{0}^{2}=\left\|\Delta^{\frac{n-1}{2}} f\right\|_{L^{2}}^{2}=\int_{\mathbb{R}^{n-1}}|\mathcal{F} f(\xi)|^{2}|\xi|^{n-1} d \xi
$$

is the continuation of (6.1) at $\mu=0$ and it vanishes on the constant function. Thus it is the unitary norm on $\mathcal{W}_{0}$. See also [24, Theorem 2.2]. Using the same proof as above we have the following result

Theorem 6.4. Let $n>2$. Then the operator $\mathcal{D}_{0, j}$ is a bounded from $\mathcal{W}_{0}$ onto $\left(\mathcal{C}_{j}, H_{1}\right)$ for $1 \leq j<\frac{n-2}{2}$. Thus $\left(\mathcal{C}_{j}, H_{1}\right), 1 \leq j<\frac{n-2}{2}$, appear as a discrete component of $\left(\mathcal{W}_{0}, H\right)_{H_{1}}$. 


\section{REFERENCES}

[1] N. Bergeron, Lefschetz properties for arithmetic real and complex hyperbolic manifolds, Int. Math. Res. Not. (2003), no. 20, 1089-1122. MR 1963482 (2004a:11040)

[2] M. Burger, J.-S. Li, and P. Sarnak, Ramanujan duals and automorphic spectrum, Bull. Amer. Math. Soc. (N.S.) 26 (1992), no. 2, 253-257. MR 1118700 (92h:22023)

[3] M. Burger and P. Sarnak, Ramanujan duals. II, Invent. Math. 106 (1991), no. 1, 1-11. MR 1123369 (92m:22005)

[4] M. Cowling, A. Dooley, A. Korányi, and F. Ricci, An approach to symmetric spaces of rank one via groups of Heisenberg type, J. Geom. Anal. 8 (1998), no. 2, 199-237.

[5] M. Engliš, S. C. Hille, J. Peetre, H. Rosengren, and G. Zhang, A new kind of Hankel type operators connected with the complementary series, Arabic J. Math. Sci. 6 (2000), 49-80.

[6] J. Faraut and A. Koranyi, Function spaces and reproducing kernels on bounded symmetric domains, J. Funct. Anal. 88 (1990), 64-89.

[7] _ Analysis on symmetric cones, Oxford University Press, Oxford, 1994.

[8] R. Howe, Transcending classical invariant theory, J. Amer. Math. Soc. 2 (1989), no. 3, 535-552.

[9] H. Jacobsen and M. Vergne, Restriction and Expansions of Holomorphic Representations, J. Funct. Anal., 34, (1979) 29-53.

[10] K. D. Johnson, Composition series and intertwining operators for the spherical principal series. II, Trans. Amer. Math. Soc. 215 (1976), 269-283.

[11] K. D. Johnson and N. R. Wallach, Composition series and intertwining operators for the spherical principal series. I, Trans. Amer. Math. Soc. 229 (1977), 137-173. MR 0447483 (56 \#5794)

[12] A. Juhl, Families of conformally covariant differential operators, Q-curvature and holography, Progress in Mathematics, vol. 275, Birkhäuser Verlag, Basel, 2009. MR 2521913 (2010m:58048)

[13] T. Kobayashi, Branching problems of unitary representations, Proc. of the International Congress of Mathematicians, Vol. II (Beijing), Higher Ed. Press, 2002, pp. 615-627.

[14] T. Kobayashi, Multiplicity-free theorems of the restrictions of unitary highest weight modules with respect to reductive symmetric pairs, Representation theory and automorphic forms, Progr. Math., vol. 255, Birkhäuser Boston, Boston, MA, 2008, pp. 45-109. MR 2369496 (2008m:22024)

[15] T. Kobayashi, F-method for construction equivariant differential operators, preprint 2012

[16] B. Kostant, On the existence and irreducibility of certain series of representations, Bull. Amer. Math. Soc. 75 (1969), 627-642. MR 0245725 (39 \#7031)

[17] J. Möllers and Y. Oshima, Restriction of complementary series representations of $O(1, N)$ to symmetric subgroups, arXiv:1209.2312/math.RT.

[18] N. Mukunda, Unitary representations of the Lorentz groups: Reduction of the supplementary series under a noncompact subgroup, J. Mathematical Phys. 9 (1968), 417-431. MR 0227317 (37 \#2902)

[19] Yu. Neretin, Plancherel formula for Berezin deformation of $L^{2}$ on Riemannian symmetric space, J. Funct. Anal. 189 (2002), no. 2, 336-408.

[20] H. Rossi and M. Vergne, Analytic continuation of the holomorphic discrete series of a semisimple Lie group, Acta Math. 136 (1976), 1-59.

[21] B. Ørsted and B. Speh, Branching laws for some unitary representations of SL(4,R), SIGMA 4 (2008).

[22] B. Speh and T. N. Venkataramana, Discrete components of some complementary series representations, Indian J. Pure Appl. Math. 41 (2010), no. 1, 145-151. MR 2650105 (2011h:22014)

[23] G. van Dijk and S. C. Hille, Canonical representations related to hyperbolic spaces, J. Funct. Anal. 147 (1997), 109-139.

[24] A. M. Vershik and M. I. Graev, The structure of complementary series and the spherical representations of the group o( $n, 1)$ and $u(n, 1)$, preprint, arXiv:math.RT/0610215.

[25] N. Wallach, The analytic continuation of the discrete series, I, II, Trans. Amer. Math. Soc. 251 (1979), $1-17 ; 19-37$.

[26] G. Zhang, Discrete components in restriction of unitary representations of rank one semisimple lie groups, arXiv:1111.6406.

[27] _ Berezin transform on real bounded symmetric domains, Trans. Amer. Math. Soc. 353 (2001), 3769-3787. 
[28] Branching coefficients of holomorphic representations and Segal-Bargmann transform, J. Funct. Anal. 195 (2002), 306-349.

[29] , Degenerate principal series representations and their holomorphic extensions, Adv. Math. 223 (2010), no. 5, 1495-1520. MR 2592500 (2011i:22013)

B. Speh, Department of Mathematics, Cornell University, NY14853-4201, USA.

EMAIL: SPEH@MATH.CORNELL.EDU

G.Zhang, Mathematical Sciences, Chalmers University of Technology and MatheMATiCAl Sciences, Göteborg University, SE-412 96 Göteborg, SWEden.

EMAIL:GENKAI@CHALMERS.SE 